University of Wollongong

Research Online

Faculty of Engineering and Information

Faculty of Engineering and Information

Sciences - Papers: Part A

Sciences

$1-1-2013$

\title{
Voltage unbalance emission assessment: sources of contribution and their ranking
}

U Jayatunga

University of Wollongong, jvupj453@uowmail.edu.au

Sarath Perera

University of Wollongong, sarath@uow.edu.au

P Ciufo

University of Wollongong, ciufo@uow.edu.au

A P. Agalgaonkar

University of Wollongong, ashish@uow.edu.au

Follow this and additional works at: https://ro.uow.edu.au/eispapers

Part of the Engineering Commons, and the Science and Technology Studies Commons

Research Online is the open access institutional repository for the University of Wollongong. For further information contact the UOW Library: research-pubs@uow.edu.au 


\title{
Voltage unbalance emission assessment: sources of contribution and their ranking
}

\begin{abstract}
etermining sources of voltage unbalance (VU) and quantifying their individual contributions in an interconnected network is a complex task due to multiple interactions that take place between them. Post-connection VU emission assessment techniques in relation to customer installations are important not only in compliance assessment according to IEC/TR 61000-3- 13:2008 Limits - Assessment of emission limits for the connection of unbalanced installations to MV, HV and EHV power systems, but also in determining the various emission contributors in the power system being considered. This paper presents a case study on the application of a recently developed post-connection VU emission assessment methodology to a $66 \mathrm{kV}$ interconnected sub-transmission network owned and operated by an Australian network service provider. The study incorporates the evaluation of constituent components of the resultant VU factors at bus bar levels, identification of the dominant emission contributors and a methodology for ranking of the emission contributions made by the asymmetrical transmission lines in the network. Outcomes obtained through the VU emission assessment methodology are validated using three-phase, unbalanced load flow analysis.
\end{abstract}

\section{Keywords}

voltage, unbalance, emission, their, assessment, ranking, sources, contribution

\section{Disciplines}

Engineering | Science and Technology Studies

\section{Publication Details}

U. Jayatunga, S. Perera, P. Ciufo \& A. P. Agalgaonkar, "Voltage unbalance emission assessment: sources of contribution and their ranking," in Australian Universities Power Engineering Conference (AUPEC 2013), 2013, pp. 1-6. 


\title{
Voltage Unbalance Emission Assessment: Sources of Contribution and their Ranking
}

\author{
U. Jayatunga, Student Member, IEEE, S. Perera, Senior Member, IEEE, P. Ciufo*, Senior Member, IEEE, \\ and A. P. Agalgaonkar, Member, IEEE \\ Australian Power Quality and Reliability Centre, \\ School of Electrical, Computer and Telecommunications Engineering, \\ University of Wollongong, Australia \\ e-mail: ppc@uow.edu.au*
}

\begin{abstract}
Determining sources of voltage unbalance (VU) and quantifying their individual contributions in an interconnected network is a complex task due to multiple interactions that take place between them. Post-connection VU emission assessment techniques in relation to customer installations are important not only in compliance assessment according to IEC/TR 61000-3 13:2008 Limits - Assessment of emission limits for the connection of unbalanced installations to MV, HV and EHV power systems, but also in determining the various emission contributors in the power system being considered. This paper presents a case study on the application of a recently developed post-connection VU emission assessment methodology to a $66 \mathrm{kV}$ interconnected sub-transmission network owned and operated by an Australian network service provider. The study incorporates the evaluation of constituent components of the resultant VU factors at busbar levels, identification of the dominant emission contributors and a methodology for ranking of the emission contributions made by the asymmetrical transmission lines in the network. Outcomes obtained through the $\mathrm{VU}$ emission assessment methodology are validated using three-phase, unbalanced load flow analysis.
\end{abstract}

Index Terms-power quality, voltage unbalance, current unbalance, voltage unbalance emission allocation, voltage unbalance emission assessment, network asymmetry, load asymmetry

\section{INTRODUCTION}

Voltage unbalance (VU) in power systems is caused by asymmetrical transmission lines and unbalanced installations [1]. However, limited knowledge is available on the determination of individual emission contributions made by different sources of unbalance [2], [3]. Further, identification of sources of VU and their level of contribution at each busbar, is a much more involved task in interconnected networks as a result of the multiple interactions that occur between many sources of unbalance [4], [5]. Thus, although the causes of VU are well known to system operators in general, their location and implementation of any corrective measures is not a trivial exercise in such interconnected networks.

Development of the VU emission allocation process given in IEC/TR 61000:3:13 [1] has made the requirement of compliance assessment a necessary step at the post connection stage to ensure that the allocated emission limits are met. The work presented in [6] and [7] has introduced new, deterministic approaches to assess constituent components of post-connection VU emission at busbar levels in radial and interconnected networks. These new approaches, which significantly extend the preliminary work covered in the CIGRE/CIRED C4.109 working group report on emission assessment techniques [9], are based on complex VU factors and utilises pre- and postconnection snap-shot based voltage/current measurements at busbar levels and known system parameters. Further, the new methodologies are sufficiently generalised such that the net VU emission at a busbar can be separated into individual contributions made by the local load asymmetry, local line asymmetries (line/s which connect the load to the neighbouring bus bars) and surrounding busbar voltage asymmetries regardless of the balanced/unbalanced nature of power system elements.

The primary objective of the present study is to apply the proposed emission assessment methodologies of interconnected networks given in [7] to a practical power system which exhibits significant unbalance levels at some of its busbars. The $66 \mathrm{kV}$ sub-transmission interconnected network under study is owned by an Australian network service provider and has to satisfy the stipulated limits [10] on maximum VU emission which is to be limited to $1 \%$. But, VU levels up to $2 \%$ at some busbars during peak demand periods have been observed in this network. The problem of VU in this network has been investigated in [4] and [8] based on a 'negative sequence VU emission vector' concept derived from [1]. The work presented in this paper investigates the VU behaviour of the system by introducing constituent components of net unbalance emissions at busbar levels and hence includes a comparison of the two approaches. Further, a new methodology has been developed to rank the influences made by asymmetrical lines based on their individual emission contributions. A threephase, unbalanced load flow program written in MATLAB is used to verify the various outcomes.

The paper is organised as follows. Section II contains a summary of the investigations and main outcomes covered in [4] and [8]. Application of the new VU emission assessment methodology is given in Section III. Methodology for ranking asymmetrical line contributions is established in Section IV. Conclusions are given in Section V.

\section{THE $66 \mathrm{KV}$ SUB TRANSMISSION NETWORK AND REVIEW OF THE PREVIOUS STUDY [4], [8]}

\section{A. Introduction to the Study System}

The $66 \mathrm{kV}$ sub-transmission network under study as shown in Fig. 1 is connected to the EHV transmission system at S1 
(bulk supply point) where the negative sequence voltage has been measured to be negligible. Some of the transmission lines of the system are longer than $50 \mathrm{~km}$ and are not systematically transposed. Levels of VU that exist at load busbars S7, S8 and S9 have been noted to reach up to $2 \%$, in addition to the significant levels $(1.2 \%)$ even at the upstream busbars S2 and S4 during the peak demand periods [4]. There was a perception that the presence of excessive VU levels are due to asymmetries associated with loads and accordingly load balancing was carried out in some busbars to address the issue. But, no significant improvements were noted directing the problem towards the location of network (line) asymmetries as sources of unbalance [8].

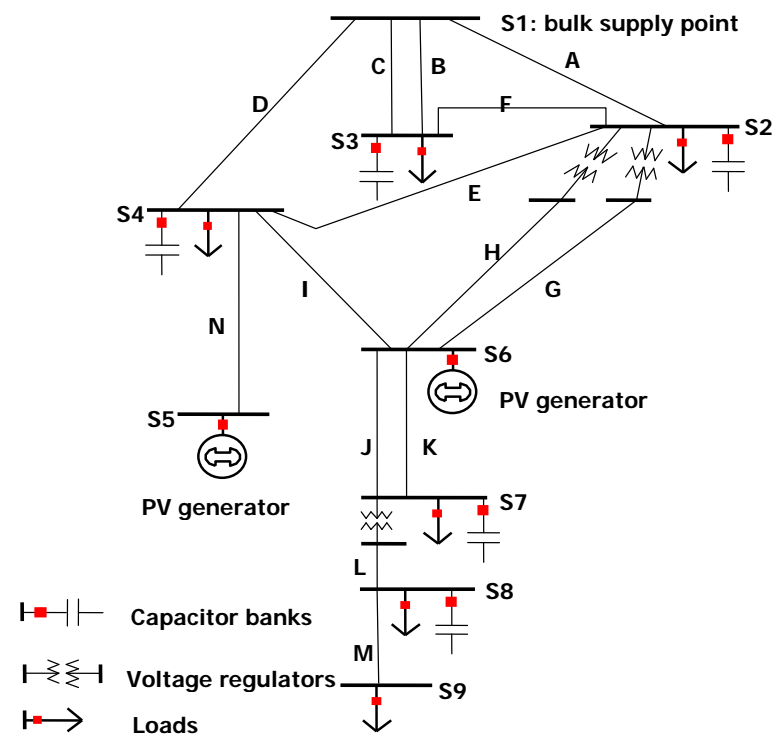

Fig. 1. $66 \mathrm{kV}$ interconnected sub transmission network

\section{B. Analysis of VU Sources and their Contributions}

The study presented in [8] evaluates the VU problem in terms of net unbalance emission at busbar levels corresponding to a selected time stamp by employing the linearity property of negative sequence variables.

In the work undertaken, the total VU emission at a busbar is not separated into its constituent components, but the net global impact on the entire network made by each source of unbalance (individual line and individual load) is considered in its entirety. In this process, the impact of line asymmetries on global VU levels at different busbars is evaluated assuming balanced loads and contributions made by load asymmetries are evaluated by assuming fully transposed transmission lines.

The VU emission made by an individual line (line under observation) is governed by the corresponding vector $Z_{-+: t} I_{+: t}$, where $Z_{-+: t}$ is the negative positive sequence coupling impedance of the line and $I_{+: t}$ is the positive sequence current flowing through that line. Conversely, the VU emission made by an individual load (load under observation) is assessed using the factor $Z_{++: t} I_{-: t}$, where $Z_{++: t}$ is the positive sequence impedance of any line which carries the negative sequence current $I_{-: t}$ caused by that load asymmetry.
The aggregated impact of the load and line asymmetries is evaluated by integrating individual emission contributions. Magnitudes of VU factors at different busbars corresponding to three different modelling scenarios are shown in Fig. 2(a) together with measured levels. Case I corresponds to the computed levels for the existing network, whereas Cases II and III correspond to (1) unbalanced lines and balanced loads and (2) balanced lines and unbalanced loads respectively. Further, this study evaluates the most dominant VU emission contributors in the network by determining the negative sequence VU emission vector for individual lines and loads against the resultant emission vector.

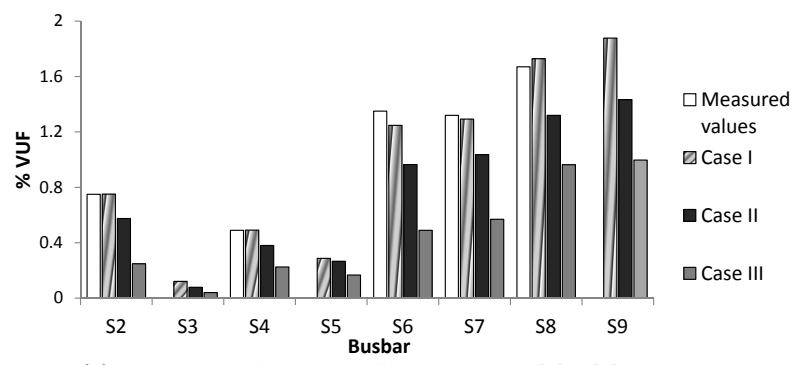

(a). Resultant VU factors at different Busbars - [2] \& [3]

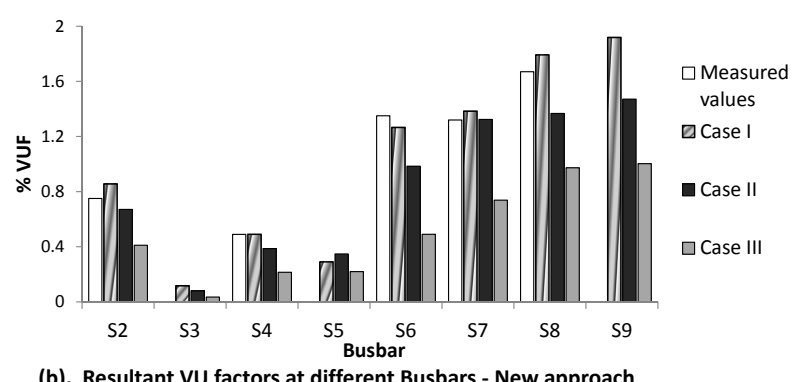

(b). Resultant VU factors at different Busbars - New approach

Fig. 2. Magnitudes of resultant VU factors at different busbars in the $66 \mathrm{kV}$ interconnected network: Case I - Original network. Case II - Network with unbalanced lines and balanced loads. Case III - Network with balanced lines and unbalanced loads.

\section{Application of the Proposed VU Emission ASSESSMENT METHODOLOGY}

A. VU Emission Assessment in Interconnected Power Systems: Summary of the Work Presented in [7]

The proposed methodology for evaluating constituent components of total VU emission at a selected busbar is far more revealing compared to the methodology described in [4], [8] as it can determine the individual emission contributors. As per the concepts used in [9], post connection VU emission for a radial network at a point of evaluation (POE) $\left(U_{2, \text { post }}\right)$ can be determined using (1).

$U_{2, \text { post }}=K \cdot U_{2, \text { pre }}+U_{2, i}=K \cdot U_{2, \text { pre }}+U_{2, i(\text { load })}+U_{2, i(\text { line })}$

where $U_{2 \text {,pre }}$ is the pre-connection VU emission at the POE; $U_{2, i}$ is the emission caused by the installation which can be expanded as the summation of unbalanced load contribution $\left(U_{2, i(\text { load })}\right)$ and asymmetrical line contribution $\left(U_{2, i(\text { line })}\right) ; K$ is a complex scaling factor. Here, the pre-connection VU emission level represents the upstream source/busbar VU emission 
in the radial network since it can be assumed that an equivalent fixed unbalanced voltage source exists at an upstream point which is not disturbed by the connection of the downstream load. Thus, the constituent components of the post-connection VU emission level at the POE in terms of VU factors given by (1), consists of load asymmetry, line asymmetry and upstream source contributions.

The methodology in [7] which is used in this paper is unique as it uses only the post connection measurements. The concept of the use of a pre-connection measurement at the POE is not applicable in the case of an interconnected network in general to take into account the contributions made by the upstream source/surrounding busbars as these busbars will be influenced by the connection of the load.

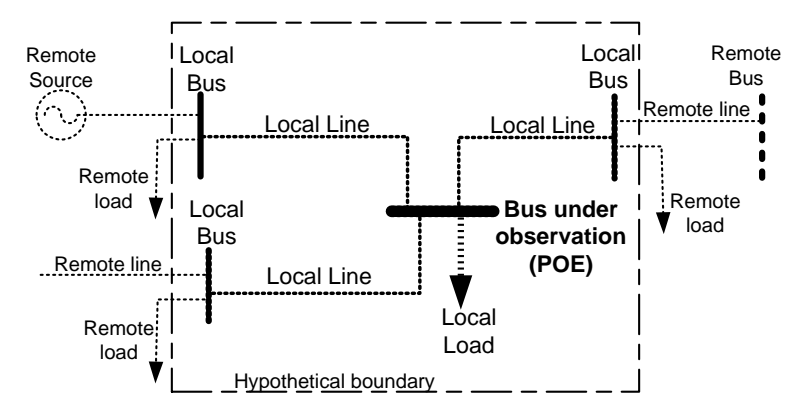

Fig. 3. Illustration of VU emission contributors in an interconnected network

As shown in Fig. 3, for the busbar under observation (POE), its emission contributions are identified as emission due to local load asymmetry, local line asymmetries and voltage asymmetries present at local busbars. In this methodology, "local" lines are defined as all lines that are connected to the busbar under observation and the local busbars are defined as all busbars which are connected to the busbar under observation through local lines.

This emission assessment formulation defines a hypothetical boundary (shown in Fig. 3) which encircles all local busbars in the network considered for decomposing the net emission level at the POE into its constituent components. The current drawn by the load at the POE affects the pre-existing busbar VU levels (except for voltage controlled busbars) due to the additional negative sequence voltage drops through lines. Local load contribution represents the influence made by load asymmetry only on the POE. Local busbars are identified as dependent VU sources which include pre-existing (preconnection) unbalance as well as the influence made by the local load on the rest of the system outside the hypothetical boundary (due to the added current to the network). The contributions made by the local load on the rest of the system outside the boundary which is embedded in the local busbar asymmetries is dependent on the level of local load unbalance and network unbalance, and is seen to be essentially the responsibility of the network owner.

Accordingly, the total VU factor at the POE $\left(V U F_{k}\right.$ for busbar ' $k$ ') is expressed as the summation of the three contributors as shown in (2) where $V U F_{\text {load, } k}$ is the contribution made by the local load connected at the POE; $V U F_{\text {line }, k}$ is the emission contribution made by all local lines; $V U F_{\mathrm{d}_{-} \text {source, } k}$ is the contribution made by local busbar voltage asymmetries. $V U F_{\mathrm{d}_{-} \text {source, } k}$ represents a dependent voltage source which is influenced by the connection of the load.

$$
V U F_{k}=V U F_{\text {load }, k}+V U F_{\text {line }, k}+V U F_{\mathrm{d}_{-} \text {source }, k}
$$

According to the theoretical formulation presented in [7], for an $n$ bus power system with passive loads (i.e. constant impedance, constant current or constant power type loads), the individual components of total $\mathrm{VU}$ emission at bus $k\left(V U F_{k}\right)$ as given in (2) is evaluated using (3), (4) and (5).

$$
\begin{gathered}
V U F_{\text {load }, k}=\sum_{i \neq k}^{n}\left(C U F_{k}-V U F_{k}\right) \frac{Y_{11: k i}}{Y_{22: k k}} V_{\text {drop-t }(k-i)} \\
V U F_{\text {line }, k}=-\sum_{i \neq k}^{n} \frac{Y_{21: k i}}{Y_{22: k k}} V_{\text {drop-t }(k-i)} \\
V U F_{\text {d_source }, k}=-\sum_{i \neq k}^{n} \frac{Y_{22: k i}}{Y_{22: k k}}\left(1+V_{\text {drop-t }(k-i)}\right) V U F_{i}
\end{gathered}
$$

where $V U F_{i}$ is the $\mathrm{VU}$ factor of busbar ' $i$ ' which is a local busbar, $C U F_{k}$ is the current unbalance factor of the local load, $V_{\text {drop-t }(k-i)}$ is the normalised (using ' $k$ th' bus voltage) positive sequence voltage drop of line ' $k-i$ ' (i.e. line connecting busbars ' $k$ ' and ' $i$ ') and $Y_{x y: i j}{ }^{1}$ represents elements of $\mathrm{Y}$ bus admittance matrix derived in sequence domain for the network ${ }^{2}$.

The above methodology can be used in practice to refine the IEC VU emission assessment guidelines by configuring an advanced power quality instrument with the proposed algorithm. It may facilitate the evaluation of VU emission as a continuous time domain function by monitoring and logging synchronised system measurements (voltage, current) and system status (generator outages, switching status of loads/lines) continuously to accommodate the changing behaviour of the power system.

\section{B. Analysis of Voltage Unbalance Behaviour of the $66 \mathrm{kV}$ Interconnected Network}

VU behaviour exhibited by the $66 \mathrm{kV}$ interconnected network is analysed by applying the new emission assessment methodology for the same time stamp as considered in [4]. The impact made by different sources of unbalance at busbar levels in terms of VU factors is established by synthesising the network with unbalanced passive loads (details are given in Appendix A) and untransposed lines (considering actual construction details of transmission lines). The resultant VU levels at different busbars $\left(V U F_{k}\right)$ and their constituent components as identified by $V U F_{\text {load }, k}, V U F_{\text {line }, k}$ and $V U F_{\text {d_source, } k}$ are given in Table I.

In general (referring to Table I), the net VU factors $\left(V U F_{k}\right)$ ( $2^{\text {nd }}$ column) is seen to be influenced to a lesser degree by the local load asymmetry ( $3^{\text {rd }}$ column) or local line asymmetries

\footnotetext{
${ }^{1} x, y$ refer to 0,1 or 2 representing zero sequence, positive sequence or negative sequence quantities respectively

${ }^{2} \mathrm{Y}$ bus admittance matrix is derived considering all three phases in the phase domain and then converted to the sequence domain
} 
TABLE I

VOLTAGE UNBALANCE EMISSION OUTCOMES OF THE $66 \mathrm{KV}$ INTERCONNECTED NETWORK: CASE I

\begin{tabular}{|l|l|l|l|l|}
\hline $\begin{array}{l}\text { Bus } \\
\#\end{array}$ & $\begin{array}{l}\text { VUF at POE } \\
V U F_{k} \%\end{array}$ & $\begin{array}{l}\text { Local load } \\
\text { contribution } \\
V U F_{\text {load }} \%\end{array}$ & $\begin{array}{l}\text { Local line } \\
\text { contribution } \\
\sum_{\%} V U F_{\text {line }}\end{array}$ & $\begin{array}{l}\text { Local busbar } \\
\text { contribution } \\
\sum_{\%} V U F_{\text {d_source }}\end{array}$ \\
\hline S2 & $0.86 \angle-154$ & $0.21 \angle 175$ & $0.04 \angle-106$ & $0.76 \angle-148$ \\
\hline S3 & $0.12 \angle-159$ & $0.02 \angle-89$ & $0.06 \angle 131$ & $0.10 \angle-134$ \\
\hline S4 & $0.50 \angle-133$ & $0.04 \angle-104$ & $0.11 \angle-12$ & $0.52 \angle-145$ \\
\hline S5 & $0.37 \angle-121$ & $0 \angle 0$ & $0.16 \angle 4.5$ & $0.49 \angle-136$ \\
\hline S6 & $1.27 \angle-160$ & $0.01 \angle 145$ & $0.16 \angle 155$ & $1.16 \angle-154$ \\
\hline S7 & $1.38 \angle-161$ & $0.25 \angle 159$ & $0.04 \angle-33$ & $1.46 \angle-155$ \\
\hline S8 & $1.80 \angle-157$ & $0.18 \angle-160$ & $0.07 \angle-3$ & $1.67 \angle-158$ \\
\hline S9 & $1.92 \angle-157$ & $0.07 \angle-131$ & $0.09 \angle-153$ & $1.77 \angle-159$ \\
\hline
\end{tabular}

( $4^{\text {th }}$ column) compared to the local busbar voltage asymmetries ( $\left.V U F_{\mathrm{d}_{\text {_source }}}\right)\left(5^{\text {th }}\right.$ column). Since the bulk supply point of the network (S1) is considered to have negligible unbalance, $V U F_{\text {d_source }}$ for a POE is primarily due to the interactive effects of asymmetries associated with the rest of the system outside the hypothetical boundary.

For the purpose of VU mitigation, it is important to investigate the dominant emission contributors in the network. In this regard, emission assessment methodology is used considering following scenarios. The integrated impact made by untransposed transmission lines only on the network was evaluated by synthesising network operation under balanced loading conditions (Case II). Conversely, the impact of unbalanced loads on the network is investigated assuming ideally transposed lines (Case III). The respective emission separation outcomes are shown in Table II. In Case III, for the system with transposed lines, $V U F_{\text {line }}$ is zero ( $8^{\text {th }}$ column$)$ as it is governed by the term $Y_{21: x y}$ which is zero for symmetrical lines. But, in Case II - system with balanced loads, small values exist for $V U F_{\text {load }}$ ( $3^{\text {rd }}$ column) since the local load contribution is governed by the negative sequence current resulting from line asymmetries. Similarly, it can be seen that the local load contribution $\left(V U F_{\text {load }}\right)$ while being small are not vastly different under both balanced and unbalanced loading conditions (columns 3 and 7). I.e. the negative sequence current flowing in the system is not significantly affected by the load unbalance. Further, significant VU improvement at busbar levels can be seen for Case III where transmission lines are fully transposed compared to the same for Case II (columns 9 and 5). Hence, the most significant emission contributors can be identified as asymmetrical lines. Fig. 2(b) illustrates the magnitudes of different busbar VU factors $\left(\left|V U F_{k}\right|\right)$ under three different scenarios discussed previously.

For each scenario, unbalanced load flow analysis in MATLAB is used to solve the given network configuration; (a) to establish the post connection voltages and currents, as well as (b) a validation technique by evaluating VU factors at busbar levels. The admittance matrix in sequence domain is also established using transmission line and capacitor bank data in order to carry out a mathematical evaluation. The following observations can be made from the study reported in this paper.

- Net VU emission at busbar levels are decomposed into constituent components in terms of VU factors as identified by load, line and local busbar voltage asymmetries in comparison to the global effect made by different sources of unbalance at busbar levels as given in [4] and [8] .

- The dominant primary emission contributors are identified as the asymmetrical transmission lines by synthesising the given network under Case II and Case III.

- The magnitudes of VU factors, as shown in Fig 2 (a) and (b) are in close agreement, thus verifying the outcomes of the new methodology against the results in [4] and [8].

\section{RANKING OF EMISSION CONTRIBUTIONS MADE BY LINE ASYMMETRIES}

As discussed in Section III-B, existing VU levels in the network are mainly caused by untransposed transmission lines. Thus, it is important to investigate the individual contributions made by various lines on the entire network as it facilitates the identification of the most influential lines and hence favourable corrective options to mitigate existing VU.

In the new emission assessment formulation, the line contribution as identified by (4) does not reflect the absolute contribution made by a particular line, but it $\left(-\frac{Y_{21: k i}}{Y_{22: k k}} V_{\mathrm{drop}-\mathrm{t}(k-i)}\right)$ represents a portion of the normalised contribution caused by all lines connected to the busbar under observation. Alternatively, referring to the VU emission separation outcomes of a radial power system as presented in [6], the contribution made by an asymmetrical line has been identified as $-\frac{Z_{21, t}}{Z_{11, t}} \frac{V_{\text {reg-line }}}{1+V_{\text {reg-line }}}$. This describes the influence made by a line which connects a source and a load together. Here, $V_{\text {reg-line }}$ is the voltage regulation of the line which is similar to the $V_{\text {drop-t }(k-i)}$ resulting from unidirectional power flow in the radial system. Thus, individual emission contribution made by a selected line (line ' $k-i$ ') in the interconnected network can be assessed by modifying the line emission in radial network as $\frac{Y_{21: k i}}{Y_{22: k i}} \frac{V_{\text {drop-t(k-i) }}}{1+V_{\text {drop-t }(\mathrm{k}-\mathrm{i})}}$ since $\frac{Y_{21: k i}}{Y_{22: k i}}=-\frac{Z_{21, t}}{Z_{11, t}}$ and $Z_{11, t}=Z_{22, t}$.

Individual line emission contributions are represented in polar plots to evaluate the most influential lines to existing unbalance. Further, summated individual line contributions are also evaluated and indicated as a resultant line contribution vector (albeit being hypothetical) alongside with individual contributions to observe the integrated effect made by asymmetrical lines on the entire network. Fig. 4(a) shows individual line contributions and the resultant contribution vector for the existing transmission network. Note that the dominant line emission contributions are only shown in the polar plot as it has been drawn to scale. Although lines F, A, I and D have significant emission levels, contribution from line $\mathrm{F}$ to the resultant line emission level is seen to be dominant as the corresponding vector lies in close proximity to the resultant vector. Therefore, the most influential line can be identified as line $\mathrm{F}$ which is to be transposed first to reduce existing unbalance. The remaining line emission contribution vectors with new resultant line emission after transposing line $\mathrm{F}$ is evaluated and plotted in Fig. 4(b). It illustrates line A as the next most influential line as the corresponding vector almost coincides with resultant line contribution vector. Similarly, 
TABLE II

VOLTAGE UNBALANCE EMISSION OUTCOMES OF $66 \mathrm{KV}$ INTERCONNECTED NETWORK: CASE II AND CASE III

\begin{tabular}{|c|c|c|c|c|c|c|c|c|}
\hline \multirow{2}{*}{\begin{tabular}{l|} 
Bus \\
$\#$
\end{tabular}} & \multicolumn{4}{|c|}{ Case II -Balanced loads, Untransposed lines } & \multicolumn{4}{|c|}{ Case III - Unbalanced loads, Ideally transposed lines } \\
\hline & $\begin{array}{l}\text { VUF at POE } \\
V U F_{k} \%\end{array}$ & $\begin{array}{l}\text { Load } \\
\text { contribution } \\
V U F_{\text {load }} \%\end{array}$ & $\begin{array}{l}\text { Line } \\
\text { contributions } \\
\sum_{\%} V U F_{\text {line }}\end{array}$ & $\begin{array}{l}\text { Local busbar } \\
\text { contributions } \\
\sum_{\%} V U F_{\mathrm{d}_{-} \text {source }}\end{array}$ & $\begin{array}{l}\text { VUF at POE } \\
V U F_{k} \%\end{array}$ & $\begin{array}{l}\text { Load } \\
\text { contribution } \\
V U F_{\text {load }} \%\end{array}$ & $\begin{array}{l}\text { Line } \\
\text { contribution } \\
\sum V U F_{\text {line }} \%\end{array}$ & $\begin{array}{l}\text { Local busbar } \\
\text { contribution } \\
\sum_{\%} V U F_{\mathrm{d}_{-} \text {source }}\end{array}$ \\
\hline S2 & $0.67 \angle-131$ & $0.06 \angle-149$ & $0.04 \angle-106$ & $0.58 \angle-130$ & $0.41 \angle 166$ & $0.15 \angle 161$ & $0 \angle 0$ & $0.26 \angle 169$ \\
\hline S3 & $0.08 \angle-163$ & $0.003 \angle-113$ & $0.06 \angle 130$ & $0.08 \angle-117$ & $0.04 \angle-151$ & $0.02 \angle-85$ & $0 \angle 0$ & $0.03 \angle-177$ \\
\hline S4 & $0.39 \angle-106$ & $0.007 \angle-88$ & $0.11 \angle-12$ & $0.40 \angle-122$ & $0.22 \angle 174$ & $0.04 \angle-110$ & $0 \angle 0$ & $0.22 \angle 165$ \\
\hline S5 & $0.35 \angle-84$ & $0 \angle 0$ & $0.16 \angle 4.5$ & $0.38 \angle-108$ & $0.22 \angle 172$ & $0 \angle 0$ & $0 \angle 0$ & $0.22 \angle 172$ \\
\hline S6 & $0.98 \angle-138$ & $0.005 \angle-175$ & $0.16 \angle 155$ & $0.93 \angle-129$ & $0.49 \angle 149$ & $0.004 \angle 83$ & $0 \angle 0$ & $0.49 \angle 150$ \\
\hline S7 & $1.33 \angle-132$ & $0.02 \angle-152$ & $0.04 \angle-33$ & $1.14 \angle-130$ & $0.74 \angle 138$ & $0.19 \angle 99$ & $0 \angle 0$ & $0.60 \angle 150$ \\
\hline S8 & $1.37 \angle-123$ & $0.09 \angle-102$ & $0.07 \angle-3$ & $1.32 \angle-127$ & $0.97 \angle 149$ & $0.15 \angle 165$ & $0 \angle 0$ & $0.83 \angle 146$ \\
\hline S9 & $1.47 \angle-125$ & $0.05 \angle-94$ & $0.09 \angle-153$ & $1.35 \angle-124$ & $1.0 \angle 149$ & $0.04 \angle-175$ & $0 \angle 0$ & $0.97 \angle 147$ \\
\hline
\end{tabular}

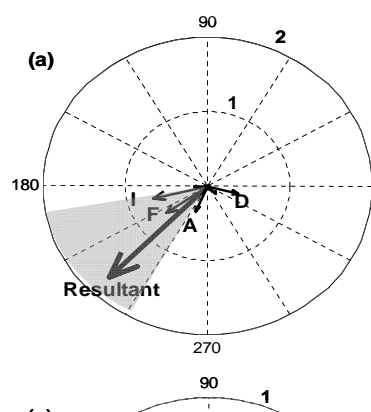

(c)
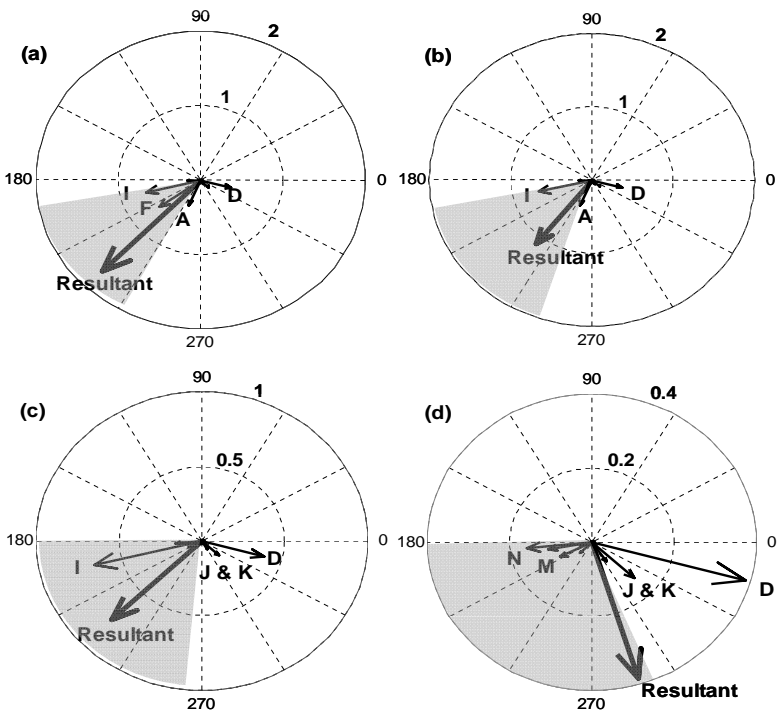

(d)

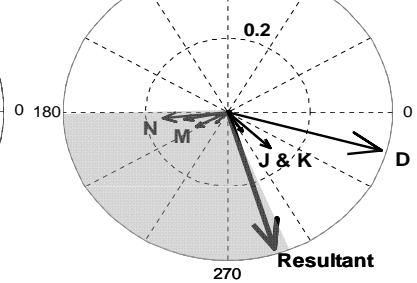

Fig. 4. Illustration of individual line contributions as \% VU factors, (a) Existing untransposed lines, (b) Line F is transposed, (c) Lines F and A are transposed, (d) Lines F, A and I are transposed

4(c) shows the next line (line I) to be transposed to improve further existing unbalance after transposing lines $\mathrm{F}$ and $\mathrm{A}$. Thus, after transposing lines F, A and I, resultant line emission contribution has reduced to $0.4 \%$ from the initial value of approximately $1.8 \%$ as shown in 4(d) and same methodology can be applied for further reduction of unbalance in terms of identification and transposition of subsequent most influential lines.

The gray sectors in each polar plot show the cluster of vectorial distribution of net $\mathrm{VU}$ emission $\left(V U F_{k}\right)$ at all busbars. Resultant line emission contribution vector is always seen to be located inside the gray sector influencing $V U F_{k}$. Thus, reduction of resultant line emission contribution vector by transposing lines reduces the busbar VU levels. These reductions of busbar VU factors prior to each line transposition exercise is clearly demonstrated by Fig. 5 .

The outcomes of this study are in close agreement with the findings of the line ranking methodology based on the analysis of emission vectors in terms of the concept of negative

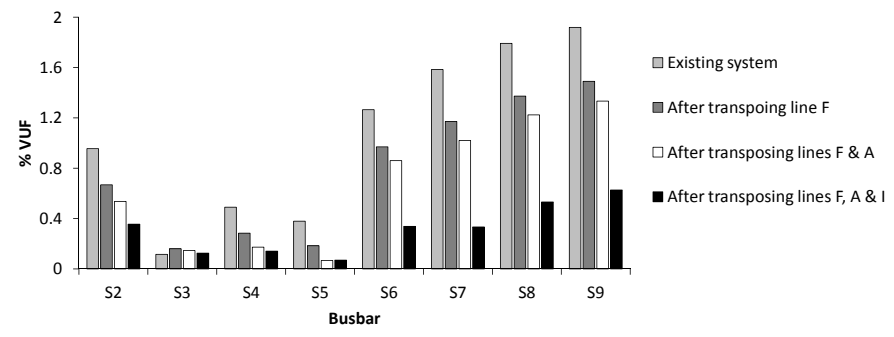

Fig. 5. Magnitudes of VU factors at different busbars with line transposition

sequence voltage drop of standalone lines as considered in [8].

\section{CONCLUSions}

The work presented in this paper covers a broad analysis of existing VU behaviour of a practical $66 \mathrm{kV}$ interconnected sub transmission network based on the recently proposed VU emission assessment methodology. Resultant VU emission at busbar levels are separated in to their constituent components as identified by contribution made by local load, contribution made by local lines and local busbar voltage asymmetry contribution. Using this approach, the most dominant emission contributors are identified as untransposed transmission lines. This paper proposes a line emission ranking methodology by evaluating individual line emission contributions which assists in determining the VU mitigation options systematically.

\section{APPENDIX A}

TABLE III

THREE PHASE UNBALANCED LOAD DISTRIBUTION OVER VARIOUS BUSBARS: EXTRACTED FROM [8]

\begin{tabular}{|l|l|l|l|l|l|l|}
\hline Load busbar & S2 & S3 & S4 & S7 & S8 & S9 \\
\hline$P_{a}(\mathrm{MW})$ & 6.32 & 6.24 & 3.87 & 11.3 & 2.04 & 0.57 \\
\hline$P_{b}(\mathrm{MW})$ & 5.87 & 6.08 & 3.43 & 11.3 & 1.89 & 0.55 \\
\hline$P_{c}(\mathrm{MW})$ & 5.87 & 5.96 & 3.37 & 11.38 & 2.04 & 0.54 \\
\hline$Q_{a} \approx Q_{b} \approx Q_{c}(\mathrm{MVAr})$ & 0.12 & 2.37 & 1.705 & 4.84 & 0.63 & 0.08 \\
\hline
\end{tabular}




\section{REFERENCES}

[1] "Electromagnetic compatibility (EMC) - limits - assessment of emission limits for the connection of unbalanced installations to $\mathrm{MV}, \mathrm{HV}$ and EHV power systems", Technical report IEC/TR 61000-3-13, Ed. 1, International Electrotechnical Commission, 2008.

[2] P. Paranavithana, S. Perera, R. Koch, and Z. Emin, "Global VU in MV Power Systems Due to Line Asymmetries", IEEE Trans. on Power Delivery, vol. 24, no. 4, pp. 2353-2360, Oct. 2009.

[3] U. Jayatunga, S. Perera and P. Ciufo, "VU management in power systems based on IEC 61000-3-13:2008: implications on the use of ' $k_{u E}$ factor", 2012 IEEE 15th International Conference on Harmonics and Quality of Power (ICHQP), pp.936,942, 17-20 June 2012.

[4] P. Paranavithana and S. Perera "Location of Sources of VU in an Interconnected Network", Proc. IEEE Power Engineering Society General Meeting, Calgary, Canada, July 2009.

[5] N.C. Woolley, J.V. Milanovic, "Statistical Estimation of the Source and Level of Voltage Unbalance in Distribution Networks", IEEE Trans. on Power Delivery, vol.27, no.3, pp.1450,1460, July 2012.

[6] U. Jayatunga, S. Perera and P. Ciufo, "Voltage unbalance emission assessment in radial power systems", IEEE Trans. on Power Delivery, vol.27, no.3, pp.1653-1661, July 2012.

[7] U. Jayatunga, S. Perera, P. Ciufo and A. P. Agalgaonkar "Voltage unbalance emission assessment in interconnected networks", accepted for publication in IEEE Trans. on Power Delivery, available at http: //works.bepress.com/pciufo/21

[8] P. Paranavithana, S. Perera, D. Sutanto and R. Koch, "A Systematic Approach Towards Evaluating Voltage Unbalance Problem in Interconnected Sub-transmission Networks: Separation of Contribution by Lines, Loads And Mitigation", Proc. 13th International Conference on Harmonics and Quality of Power, ICHQP 2008, Australia, Sept. 2008.

[9] "Review of disturbance emission assessment techniques", CIGRE/CIRED C4.109 WG Report 468, June 2011

[10] Electricity Distribution Code, Victoria, Ver. 6, January 2011 